\title{
HSD11B1 is upregulated synergistically by IFN $Y$ and TNFa and mediates TSG-6 expression in human UC-MSCS
}

\author{
Peiqing Huang (1)', Yinghong $\mathrm{Li}^{1}$, Chenchang $\mathrm{Xu}^{1}$, Gerry Melino ${ }^{2,3}$, Changshun Shao (1) ${ }^{1}$ and Yufang Shi ${ }^{1,4,5}$
}

\begin{abstract}
Inflammatory factors such as IFNY and TNFa could endow mesenchymal stem cells (MSCs) a potent immunomodulatory property, a process called licensing, but the mechanisms are not fully understood. We here found that glucocorticoid-activating enzyme 11ß-hydroxysteroid dehydrogenase type 1 (HSD11B1), which converts inactive cortisone to the active cortisol and thereby regulates tissue glucocorticoid (GC) levels, was greatly upregulated by IFNY and TNFa in human umbilical cord-derived MSCS (UC-MSCS) in a synergistic manner. While IFNY alone was not able to induce HSD11B1, it could increase the activity of NF-kB and thus augment the upregulation of HSD11B1 by TNFa. Interestingly, the upregulation of HSD11B1 by IFNY and TNFa also required glucocorticoid receptor. Furthermore, HSD11B1 was shown to be required for the expression of TNF-stimulated gene 6 (TSG-6), an important anti-inflammatory effector molecule of MSCs. Therefore, the inflammatory factors IFNY and TNFa can promote GC metabolism and thereby drive the expression of anti-inflammatory factor TSG-6 in human UC-MSCs, forming a potential negative feedback loop. These findings help to understand the relationship between inflammation and GC metabolism.
\end{abstract}

\section{Introduction}

HSD11B1 (11ß-hydroxysteroid dehydrogenase type 1), a luminally oriented enzyme of the endoplasmic reticulum membrane ${ }^{1}$, is wildly expressed in the body. It functions to convert inactive cortisone to the active cortisol ${ }^{2}$. Because endogenous glucocorticoid (GC) is indispensable for maintaining homeostasis, HSD11B1 is also expected to play a critical role in the modulation of metabolism and inflammatory response. Indeed, some chronic inflammatory conditions have been found to be associated with increased HSD11B1 expression, such as atherosclerosis ${ }^{3}$,

\footnotetext{
Correspondence: Changshun Shao (shaoc@suda.edu.cn) or

Yufang Shi (shiyufang2@gmail.com)

'State Key Laboratory of Radiation Medicine and Protection, Institute for

Translational Medicine, Key Laboratory of Stem Cells and Medical Biomaterials of Jiangsu Province, Medical College of Soochow University, Suzhou, China ${ }^{2}$ Department of Experimental Medicine, TOR, University of Rome Tor Vergata, Rome, Italy

Full list of author information is available at the end of the article

Edited by A. Rufini
}

inflammatory bowel disease, and colitis ${ }^{4,5}$. These observations are in line with many reports of induction of HSD11B1 expression by proinflammatory cytokines in various cell types ${ }^{6-8}$. In general, the regulation of HSD11B1 expression by inflammatory factors appears to be cell type-specifics. For instance, TNF $\alpha$ induce HSD11B1 expression in human fibroblasts, but not in human primary hepatocytes ${ }^{9,10}$. Therefore, the expression and function of HSD11B1 remain to be further elucidated.

Various studies have demonstrated the exciting therapeutic effect of MSCs in multiple sclerosis ${ }^{11}, \mathrm{GvHD}^{12}$, fibrosis $^{13}$, systemic lupus erythematosus (SLE) ${ }^{14}$, acute lung injury ${ }^{15}$, inflammatory bowel diseases ${ }^{16}$. And there are many ongoing Phase I-III clinical trials of MSCs in treating inflammatory disease ${ }^{17}$. However, it should be noted that MSCs are plastic in their immunomodulation $^{18}$. The immunosuppressive function of MSCs could be reversed by GC when the two combined to aggravate

\section{(c) The Author(s) 2020}

(c) (i) Open Access This article is licensed under a Creative Commons Attribution 4.0 International License, which permits use, sharing, adaptation, distribution and reproduction cc) in any medium or format, as long as you give appropriate credit to the original author(s) and the source, provide a link to the Creative Commons license, and indicate if changes were made. The images or other third party material in this article are included in the article's Creative Commons license, unless indicated otherwise in a credit line to the material. If material is not included in the article's Creative Commons license and your intended use is not permitted by statutory regulation or exceeds the permitted use, you will need to obtain permission directly from the copyright holder. To view a copy of this license, visit http://creativecommons.org/licenses/by/4.0/. 
inflammation in mice ${ }^{19}$, which reflects the complex interaction between GC and MSCs.

Published investigations have shown that the therapeutic effect of MSCs is mainly a result of immunomodulation and that this function is licensed by inflammation ${ }^{12}$. A cocktail of proinflammatory cytokines, IFN $\gamma$ in combination with any of three other cytokines, TNF $\alpha$, IL- $1 \alpha$, or IL$1 \beta$, was shown to have the capability to induce the immunosuppressive function of $\mathrm{MSCs}^{12}$. The licensed MSCs were found to secrete several factors and metabolites that mediate the anti-inflammatory effect of MSCs.

TSG-6, a hyaluronan-binding protein, is one of the factors secreted by human MSCs. It can attenuate inflammation and enhance tissue repair in mouse models of acute lung injury ${ }^{15}$, peritonitis ${ }^{20}$, myocardial infarction $^{21}$, corneal injury ${ }^{22}$, and arthritis ${ }^{23}$. However, despite the well-recognized role of TSG-6 in immunomodulation, the regulation of TSG-6 expression in human MSCs is not very clear. We previously reported that while TNF $\alpha$ could induce TSG-6 expression in human MSCs, the induction could be substantially enhanced by another cytokine IFN $\gamma$, via kynurenic acid, a metabolite of indoleamine 2,3dioxygenase (IDO) $)^{24}$.

In the present study, we found that HSD11B1 was synergistically upregulated by IFN $\gamma$ and TNFo in human umbilical cord-derived MSCs (UC-MSCs). In this process, IFN $\gamma$ was found to promote the phosphorylation of $\mathrm{p} 65$, a NF- $\mathrm{kB}$ subunit, and thus augment the HSD11B1 expression induced by TNF $\alpha$. To our surprise, glucocorticoid receptor (GR) was activated by IFN $\gamma$ and TNF $\alpha$ and mediated the upregulation of HSD11B1 by IFNY and TNF $\alpha$. We also identified HSD11B1 to be an essential molecule for the induction of anti-inflammatory TSG- 6 by IFN $\gamma$ and TNF $\alpha$. Our study reveals a novel link between inflammatory factors, HSD11B1 and TSG-6 in human UC-
MSCs. This finding increases our understanding of the regulation and function of HSD11B1 in inflammation, and may contribute to optimization of MSCs-based clinical treatments for inflammatory conditions.

\section{Results \\ HSD11B1 was synergistically upregulated by IFNp and TNFa in human UC-MSCs}

Previous reports have shown that the immunosuppressive function of MSCs is induced by proinflammatory cytokines, especially the combination of IFN $\gamma$ and TNF $\alpha$. However, most of the previously identified antiinflammatory functions of MSCs are based on the secretory effector molecules, such as TSG-6 and IGF- $2^{11}$. Because GC is critically involved in the regulation of inflammation, we set to explore the roles of GC metabolism in MSCs. Several reports showed that HSD11B1, the main enzyme that metabolizes GC, was upregulated in human fibroblasts, chondrocytes, and adipocytes by $\mathrm{TNF}^{25}$. We therefore first examined the direct effect of IFN $\gamma$ and TNF $\alpha$ stimulation on HSD11B1 expression in human UC-MSCs. We added recombinant human TNF $\alpha$ and IFNy each alone or in combination to the culture of human UC-MSCs. TNF $\alpha$ induced the expression of HSD11B1 (Fig. 1a, b), which is consistent with other studies. Surprisingly, while IFN $\gamma$ alone did not induce HSD11B1, IFN $\gamma$, and TNF $\alpha$ in combination substantially increased the expression of HSD11B1. These results showed that IFNY and TNF $\alpha$ could synergistically upregulate the expression of HSD11B1 in human UC-MSCs.

\section{NF-KB mediates the synergistic effects of IFN $\gamma$ and TNFa on HSD11B1 expression}

Since TNF $\alpha$ mainly acts through NF- $k B$ signaling in regulating downstream genes pathway, we determined the
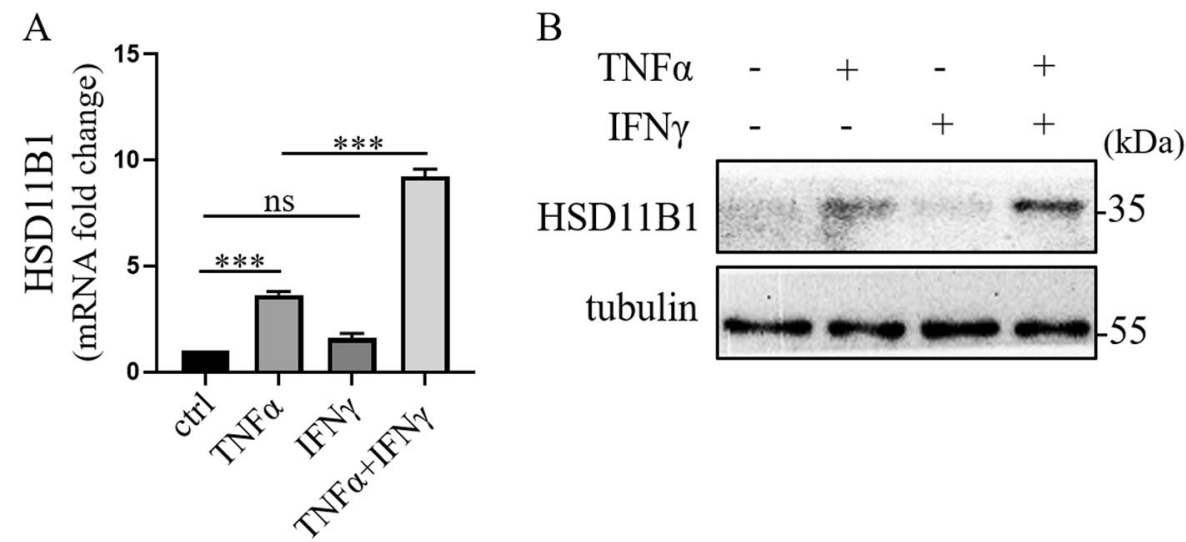

Fig. 1 The expression of HSD11B1 was significantly increased in IFNy and TNFa licensed human UC-MSCs. a Real-time PCR was employed to measure HSD11B1 in human UC-MSCs after TNFa and IFNy stimulation. The histograms represent the mean values \pm SEM from three independent experiments $(n=9)$. Asterisks indicate highly significant $\left({ }^{* * *} p<0.001\right)$ differences (Student $t$ test). Data are presented as fold change relative to ctrl human UC-MSCS. b Western blotting was performed to detect HSD11B1 in human UC-MSCS after TNFa and IFNY stimulation. 
phosphorylation level of $\mathrm{p} 65$, a NF- $\mathrm{kB}$ subunit, in human UC-MSCs in the presence of TNF $\alpha$ and IFN $\gamma$ each alone or in combination. We observed that either of the two cytokines could increase the level of p-p65, but the combination resulted in the highest p65 phosphorylation level (Fig. 2a).

To determine the role of p65 in HSD11B1 regulation, we examined the effect of BAY 11-7082, an NF- $\mathrm{kB}$ inhibitor, on HSD11B1 expression in IFN $\gamma$ and TNF $\alpha$-treated human UC-MSCs. The results showed that the upregulation of HSD11B1 by the cytokines was nearly abolished when NF- $\kappa$ B activity was inhibited (Fig. 2b, c). To further investigate this phenomenon, we knocked down p65 in human UC-MSCs by siRNA, and found that the expression of HSD11B1 was suppressed after p65 depletion (Fig. 2d, e). These results indicate that the upregulation of HSD11B1 by IFN $\gamma$ and TNF $\alpha$ was achieved through NF- $\mathrm{kB}$ signaling.

\section{GR was required for the upregulation of HSD11B1 by IFNY and TNFa}

It was well established that GCs can upregulate HSD11B1 expression in a variety of cells by activating GR.
In addition, phosphorylation at the Ser211 site was reported to be a marker of GR activation ${ }^{26}$. To test whether GR is involved in the upregulation of HSD11B1 by the two cytokines, we examined the phosphorylation level of GR in human UC-MSCs exposed to IFN $\gamma$ and TNF $\alpha$ stimulation. We found that the level of phosphorylated GR at Ser211 was significantly higher in the cytokines-treated cells than in control (Fig. 3a).

To further explore the role of GR in IFN $\gamma$ and TNF $\alpha$ induced HSD11B1 expression, we knocked down GR in human UC-MSCs by transfection with siRNA. Interestingly, after GR depletion, the upregulation of HSD11B1 by IFN $\gamma$ and TNF $\alpha$ was weakened (Fig. 3b, c), suggesting that GR is involved in the regulation of HSD11B1 by IFNY and TNF $\alpha$.

We next treated human UC-MSCs with RU486, a GC competitive GR inhibitor. As expected, RU486 greatly inhibited the induction of HSD11B1 by dexamethasone (Dex) (Fig. 3d, e). However, to our surprise, RU486 was found to promote the expression of HSD11B1 induced by IFN $\gamma$ and TNF $\alpha$ at both mRNA and protein levels (Fig. 3f, g). At the same time, level of p-GR(Ser211) increased. While RU486 inhibited the p-GR(Ser211) induced by Dex

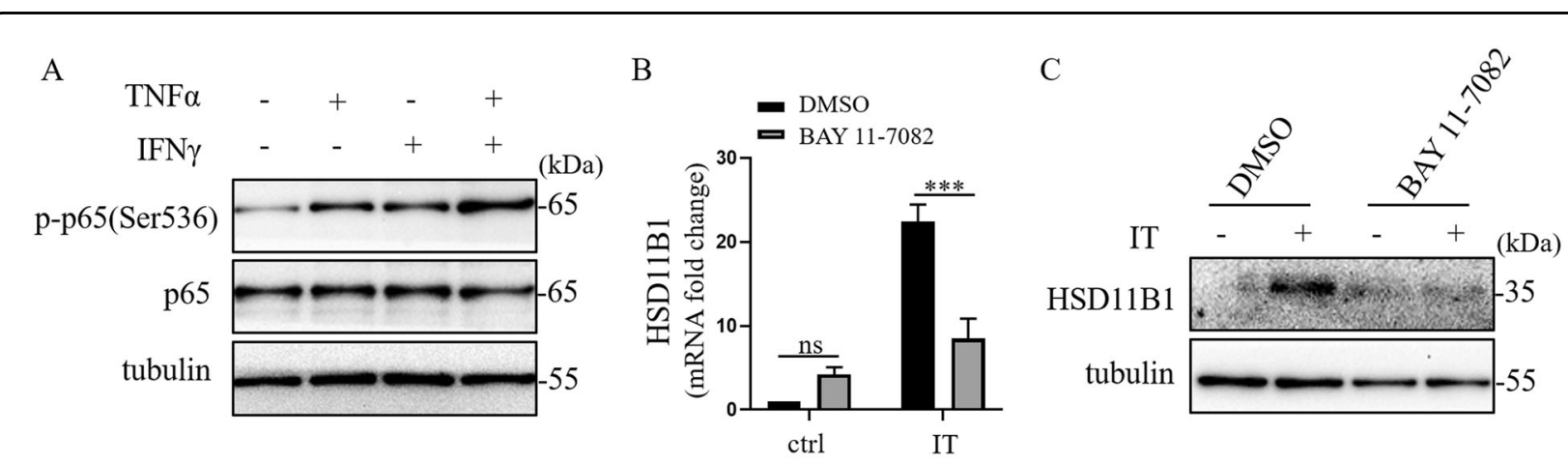

$\mathrm{D}$

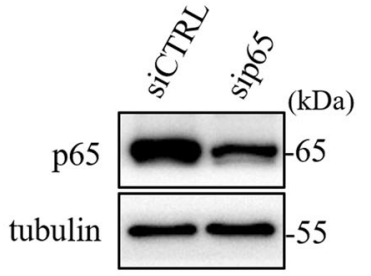

B

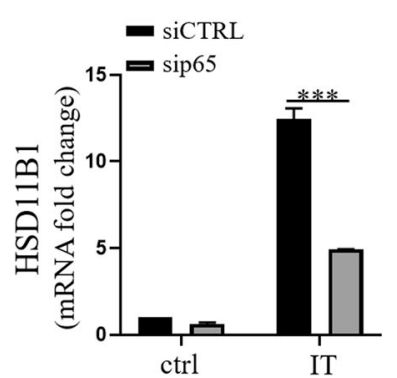

E

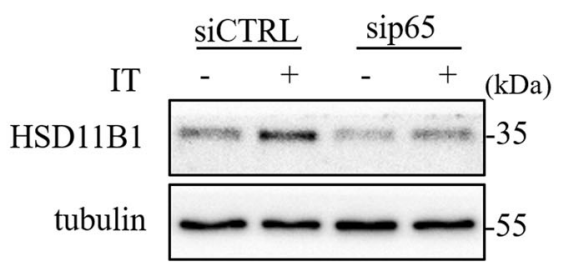

Fig. 2 IFN $\gamma$ and TNFa synergistically enhanced the expression of HSD11B1 in human UC-MSCs via NF-KB p65. a Western blotting was performed to detect p-p65 (Ser536) in human UC-MSCs after TNFa and IFNy stimulation. b Real-time PCR was employed to measure HSD11B1 in human UC-MSCs when NF-KB was inhibited by BAY 11-7082 in IT (TNFa combined with IFNץ) stimulation. The histograms represent the mean values \pm SEM from three independent experiments $(n=9)$. Asterisks indicate highly significant $\left({ }^{* * *} p<0.001\right)$ differences (ANOVA). Data are presented as fold change relative to DMSO-ctrl human UC-MSCS. c Western blotting was performed to detect HSD11B1 in human UC-MSCS when NF-KB was inhibited by BAY 11-7082 in IT stimulation. $\mathbf{d}$ Knockdown efficiency of sip65 was examined by Western blotting. Real-time PCR was employed to measure HSD11B1 in p65-depleted human UC-MSCs after IT stimulation. The histograms represent the mean values \pm SEM from three independent experiments $(n=9)$. Asterisks indicate highly significant $\left({ }^{* *} p<0.001\right)$ differences (ANOVA). Data are presented as fold change relative to siCTRL-ctrl human UC-MSCs. e Western blotting was performed to detect HSD11B1 in p65-depleted human UC-MSCs after IT stimulation. 


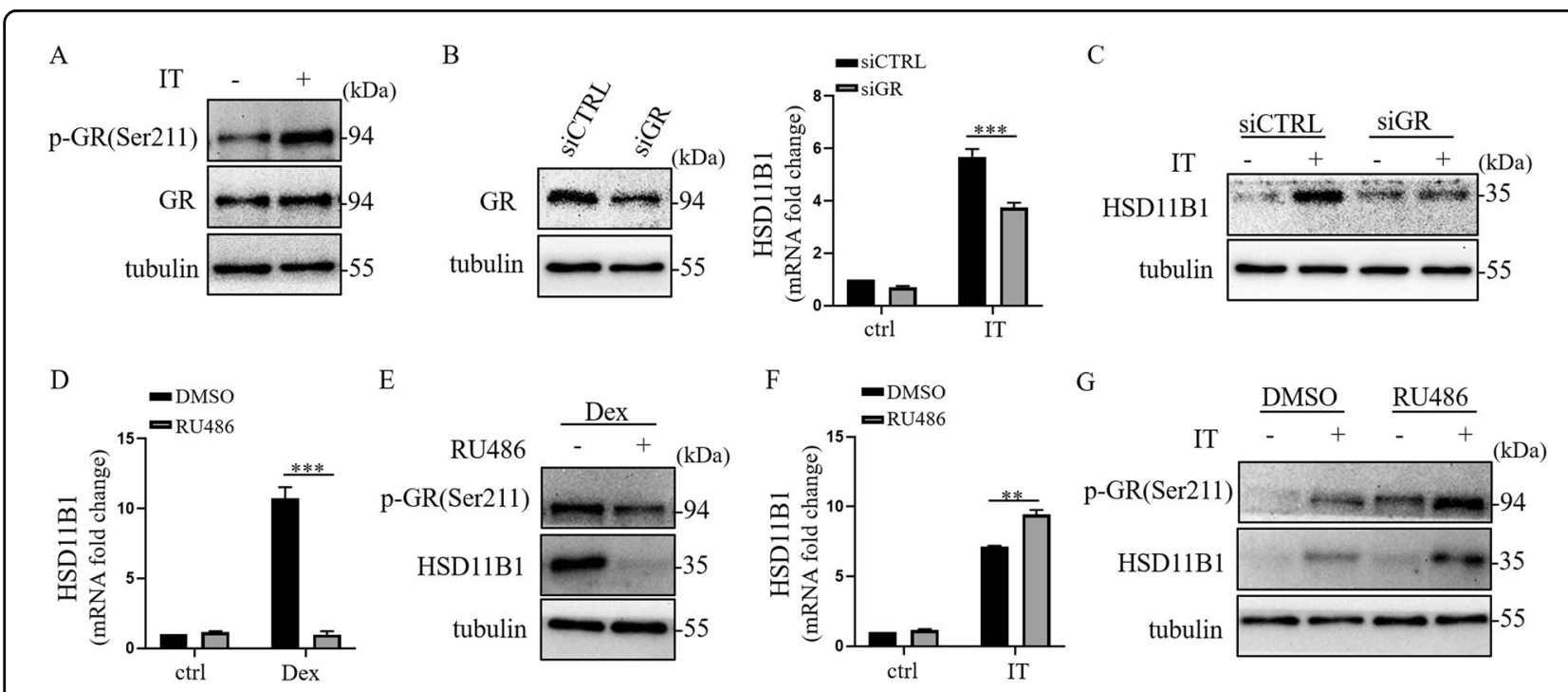

Fig. 3 Glucocorticoid receptor (GR) was involved in IFNY and TNFa-induced HSD11B1 expression. a Western blot analysis was performed to detect p-GR(Ser211) in human UC-MSCs under IT (TNFa combined with IFNY) stimulation. b Knockdown efficiency of GR was examined by Western blotting. Real-time PCR was employed to measure HSD11B1 in GR-depleted human UC-MSCs after IT stimulation. The histograms represent the mean values \pm SEM from three independent experiments $(n=9)$. Asterisks indicate highly significant $\left.{ }^{* * *} p<0.001\right)$ differences (ANOVA). Data are presented as fold change relative to siCTRL-ctrl human UC-MSCS. c HSD11B1 was detected in GR-depleted human UC-MSCs after IT stimulation by Western blotting. d Real-time PCR was performed to detect HSD11B1 in human UC-MSCs under stimulation of dexamethasone or RU486 alone or a combination of them. The histograms represent the mean values \pm SEM from three independent experiments $(n=9)$. Asterisks indicate highly significant $\left.{ }^{* * *} p<0.001\right)$ differences (ANOVA). Data are presented as fold change relative to DMSO-ctrl human UC-MSCs. e Western blotting was employed to detect HSD11B1 and p-GR(Ser211) in human UC-MSCs under stimulation of dexamethasone or RU486 alone or a combination of them. f Real-time PCR was employed to measure HSD11B1 in human UC-MSCs under stimulation of RU486 alone or a combination of IT. The histograms represent the mean values \pm SEM from three independent experiments $(n=9)$. Asterisks indicate highly significant $\left({ }^{* *} p<0.01\right)$ differences $($ ANOVA). Data are presented as fold change relative to DMSO-ctrl human UC-MSCs. g Western blotting was employed to detect HSD11B1 and p-GR(Ser211) in human UC-MSCs under stimulation of RU486 alone or a combination of IT.

(Fig. 3e), it increased the level of p-GR(Ser211) induced by IFNY and TNF $\alpha$ (Fig. 3g). These results indicated that while RU486 can competitively inhibit the effect of Dex, it does not impair the function of GR in promoting HSD11B1 expression in the context of IFN $\gamma$ and TNF $\alpha-$ treated human UC-MSCs. This result suggest that GR probably played a structural role in IFN $\gamma$ and TNF $\alpha-$ licensed human UC-MSCs. Future studies are required to determine whether GR functions to coordinate the binding of NF-kB and other transcription factors to HSD11B1 promoter.

\section{HSD11B1 is required for the induction of TSG- 6 by IFN $\gamma$ and TNFa}

Like that of HSD11B1, the expression of TSG-6, an important immunoregulatory molecule, was significantly increased when stimulated by IFN $\gamma$ and TNF $\alpha$ (Fig. 4a). We next tested whether there is link between HSD11B1 and TSG- 6 by depleting HSD11B1 in human UC-MSCs. Interestingly, the expression of TSG- 6 was greatly reduced after HSD11B1 depletion in IFN $\gamma$ and TNF $\alpha$ licensed human UC-MSCs (Fig. 4b), which strongly suggested that HSD11B1 was involved in the upregulation of TSG- 6 by IFN $\gamma$ and TNF $\alpha$.
TSG-6 was identified as a transcriptional target of NF$\kappa B^{27}$. As expected, the expression of TSG-6 in human UCMSCs was downregulated when treated with NF- $\mathrm{KB}$ inhibitor, BAY 11-7082 (Fig. 4c). Furthermore, the phosphorylation of p65 in HSD11B1-depleted human UCMSCs was decreased (Fig. 4d), which indicates that HSD11B1 may contribute to the maintenance of p65 signal pathway. Taken together, the data suggested that HSD11B1 might mediate the expression of TSG-6 through NF-kB.

\section{Discussion}

The MSCs-based treatment of inflammatory diseases is being widely tested in preclinical and clinical studies. Because of their powerful anti-inflammatory property, it is safe to predict that MSCs therapy will be a potential treatment for diseases such as SARS and the current COVID-19, or novel coronavirus pneumonia, in which the damage caused by cytokine storms is hard to control. Previous studies had demonstrated that the immunosuppressive ability of MSCs is not innate, but, is induced by the proinflammatory cytokines, like IFNY in combination with $\mathrm{TNF}^{12}$. Thus, ironically, proinflammatory cytokines can lead to immunosuppression under certain 
A

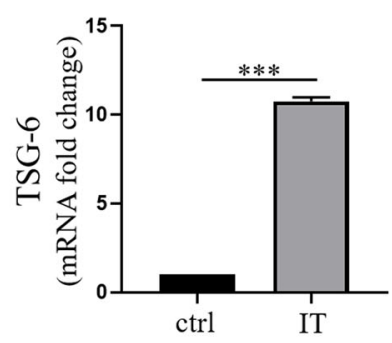

C

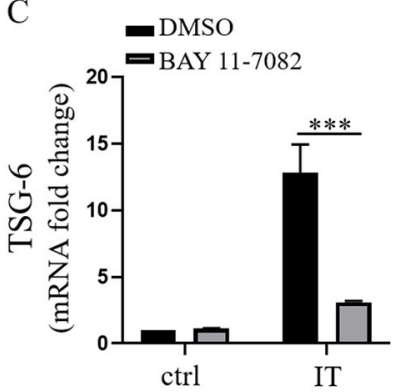

B

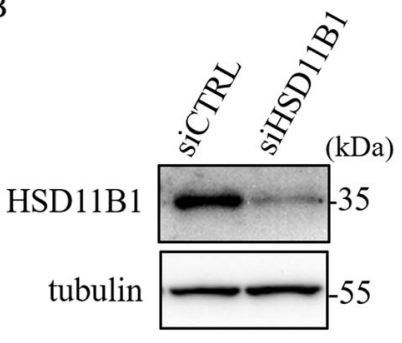

D

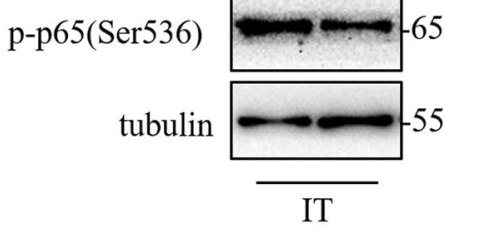

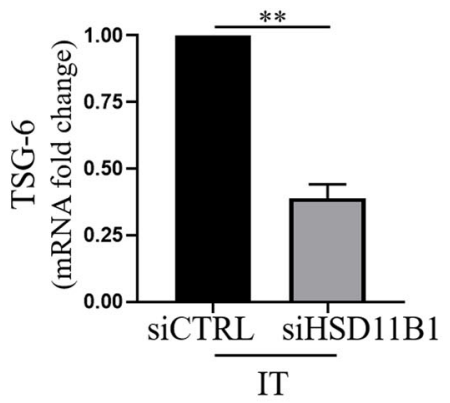

E

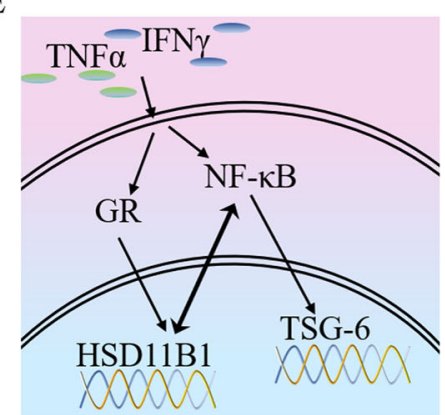

Fig. 4 HSD11B1 mediated the expression of TSG-6 induced by IFN $\boldsymbol{y}$ and TNFa via NF-kB. a TSG-6 in human UC-MSCS under IT (TNFa combined with IFNY) stimulation was detected by real-time PCR. The histograms represent the mean values \pm SEM from three independent experiments $(n=9)$. Asterisks indicate highly significant $\left({ }^{* *} p<0.001\right)$ differences (Student' $t$ test). Data are presented as fold change relative to ctrl human UC-MSCs. b Knockdown efficiency of siHSD11B1 was examined by Western blotting. TSG-6 in HSD11B1-depleted human UC-MSCs after IT stimulation was measured by real-time PCR. The histograms represent the mean values \pm SEM from three independent experiments ( $n=9$ ). Asterisks indicate highly significant $\left({ }^{* *} p<0.01\right)$ differences (Student' $t$ test). Data are presented as fold change relative to siCTRL human UC-MSCS. c TSG-6 in human UC-MSCs under BAY 11-7082 and IT stimulation was analyzed by real-time PCR. The histograms represent the mean values \pm SEM from three independent experiments $(n=9)$. Asterisks indicate highly significant $\left({ }^{* *} p<0.001\right)$ differences (ANOVA). Data are presented as fold change relative to DMSO-ctrl human UC-MSCs. d p-p65(Ser536) in HSD11B1-depleted human UC-MSCs under IT stimulation was detected by Western blotting. e The schematic overview of IFNy and TNFa regulated HSD11B1 production and HSD11B1 regulated TSG-6 production in human UC-MSCS.

circumstances $^{12}$. In human UC-MSCs, these cytokines induce a dramatic upregulation in HSD11B1, an important enzyme that metabolizes and activates GC, which may enhance local anti-inflammatory effects of GC.

Stimulation of HSD11B1 activity following TNF $\alpha$ exposure has been described in early progenitor cells of the mesenchymal lineage and differentiated cells such as osteoblasts and adipocytes, particularly in cells of human origin $^{26,28,29}$. These studies concluded that HSD11B1 induction by TNF $\alpha$ may enhance local GC generation and function. The regulation of HSD11B1 expression by IFN $\gamma$ is less understood, although. IFN $\gamma$ was reported to inhibit HSD11B1 expression in monocytes ${ }^{30}$. To our surprise, we observed that while IFNy alone was unable to induce HSD11B1, it synergistically increased the expression of HSD11B1 when combined with TNFa. Our results thus revealed that the upregulation of HSD11B1 is exerted through the concerted action of TNF $\alpha$ and IFN $\gamma$ in human UC-MSCs.

NF- $\mathrm{kB}$ was reported to positively regulate HSD11B1 expression in human synovial fibroblasts and human dermal fibroblasts ${ }^{31}$ and in adipocytes of diet-induced obese mice $^{32}$. However, it was also shown to inhibit the expression of HSD11B1 in mouse skeletal muscle cells ${ }^{33}$. Our results with human UC-MSCs are consistent with those obtained with fibroblasts and adipocytes, which are presumably derived from MSCs. It should be noted that $\mathrm{C} / \mathrm{EBP} \alpha$ and $\mathrm{C} / \mathrm{EBP} \beta$ also serve as positive regulators of HSD11B1 P1 promoter regulator ${ }^{34}$. It is therefore reasonable to speculate that $\mathrm{C} / \mathrm{EBP}$ may be involved in HSD11B1 regulation by IFN $\gamma$ and TNF $\alpha$. Further studies may reveal more species-, tissue- and context-specific factors in the regulation of HSD11B1.

GC is well documented to stimulate HSD11B1 expression and activity in most cell types. Consistently, HSD11B1 was found to be greatly upregulated by dexamethasone in human UC-MSCs, and this effect was abolished when RU486, a GC competitive GR inhibitor, was applied. Surprisingly, the HSD11B1 upregulation by IFN $\gamma$ and TNF $\alpha$ was even further increased by RU486. In contrast, GR depletion led to a suppression of HSD11B1 in the same context. These seemingly contradictory results could be due to differential consequences of RU486 treatment and GR depletion. RU486 binding to 
GR may induce some conformational change of GR and further activate it. Indeed, the phosphorylation of GR at the Ser211, a marker of GR activation, was enhanced by RU486, which might lead to augmented HSD11B1 expression. These data collectively suggest that GR may play a structural role in the upregulation of HSD11B1 by IFN $\gamma$ and TNF .

We found that the upregulation of HSD11B1 by the inflammatory factors in human UC-MSCs contributes to their anti-inflammatory function. Depletion of HSD11B1 dramatically reduced the expression of TSG-6, suggesting that HSD11B1 is required for MSCs to acquire some of their anti-inflammatory properties.

Previous studies suggested that increasing HSD11B1 expression is a mechanism to initiate the process of inflammatory resolution through increased local GC generation in IL1 $\beta$ and lipopolysaccharide stimulation ${ }^{35}$. However, blocking GC binding to GR did not decrease the expression of TSG- 6 induced by TNF $\alpha$ and IFN $\gamma$ stimulation (data not show), which suggested that HSD11B1 could upregulated TSG-6 independent of GC. We confirmed TSG-6 as a target gene of NF- $\mathrm{kB}$ in human UCMSCs. Furthermore, we noticed that the activity of NF- $\mathrm{kB}$ was repressed in HSD11B1-depleted MSCs. These results suggested that HSD11B1 mediated the induction of TSG6 through NF-kB (Fig. 4e). The feedforward loop between NF- $\mathrm{KB}$ and HSD11B1 may augment the immunosuppressive function of MSCs under inflammatory conditions.

In conclusion, we demonstrated a critical role for GC metabolism in the acquisition of the immunosuppressive capability by human UC-MSCs exposed to proinflammatory cytokines. These findings may allow the better clinical utilization of MSCs.

\section{Materials and methods}

Isolation, expansion, and cultivation of human UC-MSCs

The human umbilical cord was obtained from the First Affiliated Hospital of Soochow University with the informed consent of the donor and approved for use by the ethical standards of the Ethics Committee of both Soochow University and the First Affiliated Hospital of Soochow University.

Human UC-MSCs were obtained from human umbilical cord using a previously described protocol ${ }^{15}$. Briefly, the umbilical cord was stored in sterilized glass bottles containing phosphate-buffered saline (PBS) for processing within $4 \mathrm{~h}$. Then the umbilical cord was washed with PBS to remove blood, and then minced and seeded into cell culture dishes with low-glucose DMEM (HyClone, Neb, USA)-containing 10\% fetal bovine serum (FBS) (Gibco, MA, USA), penicillin/streptomycin (10 units) (Thermo Fisher Scientific, MA, USA), and were incubated at $37^{\circ} \mathrm{C}$ in $5 \% \mathrm{CO}_{2}$ atmosphere. The medium was replaced for every $48 \mathrm{~h}$ with fresh medium. Upon reaching $80-90 \%$ confluency, cells were harvested using trypsin-EDTA and then used for experiments immediately or frozen and stored in liquid nitrogen. Human UC-MSCs were identified by the specific cell surface markers, including HLADR (-), CD45 (-), CD31 (-), CD34 (-), CD105 (+), CD29 (+), CD90 (+), CD73 (+), and CD44 (+). These MSCs were capable of differentiating into osteocytes and adipocytes under the respective differentiation conditions.

Human UC-MSCs were maintained in low-glucose DMEM (HyClone, USA), supplemented with FBS (10\%) (Gibco, USA), penicillin/streptomycin (10 units) (Thermo Fisher, USA), $10 \mathrm{ng} / \mathrm{ml}$ human-bFGF (R\&D, USA) and incubated at $37^{\circ} \mathrm{C}$ in the presence of $5 \% \mathrm{CO}_{2}$. The media were replaced for every $72 \mathrm{~h}$ and the cells were split twice a week. All cells were regularly tested to ensure they were mycoplasma-free. Cells were used before the 12th passage.

\section{Treatments of human UC-MSCs}

Human UC-MSCs were cultured in 12-well plates, upon reaching $80-90 \%$ confluency, cells were washed with PBS and then treated with indicated stimulations. The stimulations were TNF $\alpha$ (eBioscience, MA, USA) or IFNY (eBioscience, USA) alone or a combination of these two cytokines $(10 \mathrm{ng} / \mathrm{ml}$ each) for $24 \mathrm{~h}$; TNF $(10 \mathrm{ng} / \mathrm{ml})$ and IFNY $(10 \mathrm{ng} / \mathrm{ml})$ combined with BAY 11-7082 $(5 \mu \mathrm{M})$ (Selleck, WA, USA) or DMSO (Sigma, MA, USA) for $24 \mathrm{~h}$; TNFa $(10 \mathrm{ng} / \mathrm{ml})$ and IFNY $(10 \mathrm{ng} / \mathrm{ml})$ combined with RU486 (3 $\mu \mathrm{M})$ (Selleck, USA) or DMSO for $24 \mathrm{~h}$; Dexamethasone $(10 \mathrm{ng} / \mathrm{ml})$ (Sigma, USA) combined with RU486 $(3 \mu \mathrm{M})$ or DMSO for $24 \mathrm{~h}$.

\section{Real-time PCR}

Total RNA was extracted from cell lysates according to the manufacturer's instructions using Trizol reagent (Thermo Fisher Scientific, USA). First-strand cDNA synthesis was performed using PrimeScript ${ }^{\mathrm{Tm}}$ RT Master Mix (TaKaRa Biotech, Dalian, China) according to the manufacturer's instructions. The total reaction volume of $10 \mu \mathrm{L}$ was comprised of $1 \mathrm{ng}$ cDNA, $3 \mu \mathrm{L}$ DNAase/ RNAse-free water (TaKaRa Biotech, China), $1 \mu \mathrm{L}$ primers (GENEWIZ, Suzhou, China), $5 \mu$ L SYBR qPCR SuperMix plus (with ROX) (Novoprotein, Shanghai, China). After pipetting the reaction mixture into a 384-well plate, realtime PCR was running by QuantStudio 6 Flex (Applied Biosystems, MA, USA). mRNA levels were calculated referring to $\beta$-actin as a housekeeping gene. To compare the expression of each gene in the different treatment conditions, the fold change of expression was calculated using the equation $2^{-\Delta \Delta \mathrm{Ct}}$ where $\Delta \Delta \mathrm{Ct}=\Delta \mathrm{Ct}$ (treated) $\Delta \mathrm{Ct}$ (control), $\Delta \mathrm{Ct}$ (treated) $=[\mathrm{Ct}$ (target gene) $-\mathrm{Ct}$ $(\beta$-actin $)], \quad \Delta \mathrm{Ct} \quad($ control $)=[\mathrm{Ct} \quad($ target gene $)-\mathrm{Ct}$ $(\beta$-actin)]. Real-time PCR was performed using the 
following primers $\beta$-actin-F $5^{\prime}$-TTGCCGACAGGAT GCAGAAGGA-3', $\beta$-actin-R $5^{\prime}$-AGGTGGACAGCGA GGCCAGGAT-3', HSD11B1-F 5'-AGCAGGAAAGCT CATGGGAG-3', HSD11B1-R 5'-CCACGTAACTGAG GAAGTTGAC-3', TSG-6-F 5'-TTTCTCTTGCTATGG GAAGACAC-3', TSG-6-R 5'-GAGCTTGTATTTGC CAGACCG-3'. The total amount of mRNA was normalized to endogenous $\beta$-actin mRNA.

\section{Western blotting}

Total cellular proteins were extracted by RIPA buffer (Beyotime, Shanghai, China) containing PMSF (Beyotime, China) and phosphatase inhibitors (Roche, NJ, USA). Protein samples were separated on a $10 \%$ sodium dodecyl sulfate-polyacrylamide gel, and separated proteins were electroblotted onto polyvinylidene difluoride membranes. The membranes were blocked for $2 \mathrm{~h}$ with tris-buffered saline containing Tween 20 (TBST) with 5\% albumin bovine serum (Amresco, OH, USA) at room temperature with gentle shaking. Then incubated the membranes with primary antibodies HSD11B1 (ab39364, abcam, MA, USA), tubulin (2128S, Cell Signaling Technology, MA, USA), p-p65 (Ser536) (3033S, Cell Signaling Technology, USA), p65 (8242S, Cell Signaling Technology, USA), pGR (Ser211) (4161S, Cell Signaling Technology, USA), or GR (12041S, Cell Signaling Technology, USA) for $10 \mathrm{~h}$ at $4{ }^{\circ} \mathrm{C}$ with gently shaking. After washing 3 times with TBST for $5 \mathrm{~min}$ each, the membranes were incubated with horse radish peroxidase-conjugated rabbit secondary antibodies (7074S, Cell Signaling Technology, USA) for $1 \mathrm{~h}$ at room temperature with gentle shaking. After incubation, membranes were harvested with NcmECL Ultra Kit (NCM biotech, Suzhou, China) according to the manufacturer's instructions and the signal was detected by Ultra-sensitive automatic imaging analysis system (ProteinSimple, CA, USA). Tubulin was used as an internal control.

\section{Transfections with siRNA}

Human UC-MSCs were plated on 12-well plate at the density of $50 \%$ per well and were treated with $1 \mu \mathrm{l}$ INTERFERin $^{\circledast}$ reagent (PolyPlus-transfection, Illkirch, France) along with $1 \mu \mathrm{l}$ GR, p65 or HSD11B1 siRNA (GenePharma, Shanghai, China). The same amount of control siRNA (GenePharma, China) was also transfected into MSCs as control according to the manufacturer's protocols. After $48 \mathrm{~h}$, cells were washed twice with PBS, and then cells were treated with indicated stimulations. The efficiency of transection was monitored by Western blotting. p65 siRNA sequences were 5'-CCCUAUCCC UUUACGUCAUTT-3' ${ }^{\prime}$ and $5^{\prime}$-AUGACGUAAAGGGA UAGGGTT-3'. GR siRNA sequences were $5^{\prime}$-GAUG UAAGCUCUCCUCCAUTT- $3^{\prime}$ and $5^{\prime}$-AUGGAGGAG AGCUUACAUCTT-3'. HSD11B1 siRNA sequences were
5'-GCAGGAAGAUCCUGGAAUUTT- ${ }^{\prime}$ and $5^{\prime}$-AAUUC CAGGAUCUUCCUGCTT- $3^{\prime}$. Control siRNA sequences were $5^{\prime}$-UUCUCCGAACGUGUCACGUTT- $3^{\prime}$ and $5^{\prime}$-AC GUGACACGUUCGGAGAATT-3'.

\section{Statistical analysis}

Each experiment was performed in biological triplicate each time and repeated independently at least three times. The data are presented as mean \pm standard error of the mean. Statistical significance was analyzed using Student's $t$ test, analysis of variance test calculation using GraphPad Prism 8 (GraphPad Software Inc., CA, USA), $p$ values $<0.05$ were considered statistically significant. $p<0.05$ is denoted as *, $p<0.01$ as ${ }^{* * *}, p<0.001$ as ${ }^{* * * *}, p<$ 0.0001 as $\%$ \%

\section{Acknowledgements}

We are grateful to Lijuan Cao, Liying Zhang and Ya'nan Li for providing human UC-MSCs. This study was supported by grants from the National Key R\&D Program of China [2018YFA0107500], National Natural Science Foundation of China [81530043, 81930085, and 31771260], the Scientific Innovation Project of the Chinese Academy of Sciences [XDA16020403], the Social Development Project of Jiangsu Province [BE2016671], and the State Key Laboratory of Radiation Medicine and Protection, Soochow University [GZN1201804 and GZN1201903].

\section{Author details}

${ }^{1}$ State Key Laboratory of Radiation Medicine and Protection, Institute for Translational Medicine, Key Laboratory of Stem Cells and Medical Biomaterials of Jiangsu Province, Medical College of Soochow University, Suzhou, China. ${ }^{2}$ Department of Experimental Medicine, TOR, University of Rome Tor Vergata, Rome, Italy. ${ }^{3}$ Medical Research Council (MRC) Toxicology Unit, University of Cambridge, Cambridge, UK. ${ }^{4}$ The First Affiliated Hospital of Soochow University, Suzhou, China. ${ }^{5}$ Key Laboratory of Tissue Microenvironment and Tumor, Shanghai Institutes for Biological Sciences, Chinese Academy of Sciences, Shanghai, China

\section{Competing interests}

The authors declare no competing interests.

\section{Publisher's note}

Springer Nature remains neutral with regard to jurisdictional claims in published maps and institutional affiliations.

Received: 22 March 2020 Revised: 2 April 2020 Accepted: 5 April 2020 Published online: 20 April 2020

\footnotetext{
References

1. Friedman, E. M. \& Irwin, M. Central CRH suppresses specific antibody responses: effects of beta-adrenoceptor antagonism and adrenalectomy. Brain Behav. Immun. 15, 65-77 (2001).

2. Stewart, P. M. Tissue-specific Cushing's syndrome, 11beta-hydroxysteroid dehydrogenases and the redefinition of corticosteroid hormone action. Eur. J. Endocrinol. 149, 163-168 (2003).

3. Hermanowski-Vosatka, A. et al. 11 beta-HSD1 inhibition ameliorates metabolic syndrome and prevents progression of atherosclerosis in mice. J. Exp. Med. 202, 517-527 (2005).

4. Stegk, J. P., Ebert, B., Martin, H. J. \& Maser, E. Expression profiles of human 11 beta-hydroxysteroid dehydrogenases type 1 and type 2 in inflammatory bowel diseases. Mol. Cell Endocrinol. 301, 104-108 (2009).

5. Zbankova, S. et al. 11 beta-hydroxysteroid dehydrogenase 1 and 2 expression in colon from patients with ulcerative colitis. J. Gastroenterol. Hepatol. 22, 1019-1023 (2007).
} 
6. Cai, T. Q. et al. Induction of 11 beta-hydroxysteroid dehydrogenase type 1 but not -2 in human aortic smooth muscle cells by inflammatory stimuli. J. Steroid Biochem. Mol. Biol. 77, 117-122 (2001).

7. Cooper, M. S. et al. Modulation of 11beta-hydroxysteroid dehydrogenase isozymes by proinflammatory cytokines in osteoblasts: an autocrine switch from glucocorticoid inactivation to activation. J. Bone Miner. Res. 16, 1037-1044 (2001)

8. Kaur, K. et al. Synergistic induction of local glucocorticoid generation by inflammatory cytokines and glucocorticoids: implications for inflammation associated bone loss. Ann. Rheum. Dis. 69, 1185-1190 (2010).

9. Hardy, R. S. et al. Differential expression, function and response to inflammatory stimuli of 11 beta-hydroxysteroid dehydrogenase type 1 in human fibroblasts: a mechanism for tissue-specific regulation of inflammation. Arthritis Res. Ther. 8, R108 (2006).

10. Iwasaki, Y. et al. Is the metabolic syndrome an intracellular Cushing state? Effects of multiple humoral factors on the transcriptional activity of the hepatic glucocorticoid-activating enzyme (11beta-hydroxysteroid dehydrogenase type 1) gene. Mol. Cell Endocrinol. 285, 10-18 (2008).

11. Du, L. et al. IGF-2 preprograms maturing macrophages to acquire oxidative phosphorylation-dependent anti-inflammatory properties. Cell Metab. 29, 1363-1375. e1368 (2019).

12. Ren, G. W. et al. Mesenchymal stem cell-mediated immunosuppression occurs via concerted action of chemokines and nitric oxide. Cell Stem Cell 2, 141-150 (2008).

13. Ortiz, L. A. et al. Interleukin 1 receptor antagonist mediates the antiinflammatory and antifibrotic effect of mesenchymal stem cells during lung injury. Proc. Natl Acad. Sci. USA 104, 11002-11007 (2007).

14. Wang, D. et al. A long-term follow-up study of allogeneic mesenchymal stem/ stromal cell transplantation in patients with drug-resistant systemic lupus erythematosus. Stem Cell Rep. 10, 933-941 (2018).

15. Bruscoli, S. et al. Lack of glucocorticoid-induced leucine zipper (GILZ) deregulates B-cell survival and results in B-cell lymphocytosis in mice. Blood $\mathbf{1 2 6}$ 1790-1801 (2015).

16. Soontararak, S. et al. Mesenchymal stem cells (MSC) derived from induced pluripotent stem cells (iPSC) equivalent to adipose-derived MSC in promoting intestinal healing and microbiome normalization in mouse inflammatory bowel disease model. Stem Cells Transl. Med. 7, 456-467 (2018).

17. Shi, Y. et al. Immunoregulatory mechanisms of mesenchymal stem and stromal cells in inflammatory diseases. Nat. Rev. Nephrol. 14, 493-507 (2018).

18. Wang, Y., Chen, X. D., Cao, W. \& Shi, Y. F. Plasticity of mesenchymal stem cells in immunomodulation: pathological and therapeutic implications. Nat Immunol. 15, 1009-1016 (2014).

19. Chen, $X$. et al. The interaction between mesenchymal stem cells and steroids during inflammation. Cell Death Dis. 5, e1009 (2014).
20. Choi, H., Lee, R. H., Bazhanov, N., Oh, J. Y. \& Prockop, D. J. Anti-inflammatory protein TSG-6 secreted by activated MSCs attenuates zymosan-induced mouse peritonitis by decreasing TLR2/NF-kappaB signaling in resident macrophages. Blood 118, 330-338 (2011).

21. Lee, R. H. et al. Intravenous hMSCs improve myocardial infarction in mice because cells embolized in lung are activated to secrete the anti-inflammatory protein TSG-6. Cell Stem Cell 5, 54-63 (2009).

22. Oh, J. Y. et al. Anti-inflammatory protein TSG-6 reduces inflammatory damage to the cornea following chemical and mechanical injury. Proc. Natl Acad. Sci. USA 107, 16875-16880 (2010).

23. Mindrescu, $C$. et al. Reduced susceptibility to collagen-induced arthritis in DBA1J mice expressing the TSG-6 transgene. Arthritis Rheum. 46, 2453-2464 (2002).

24. Wang, G. et al. Kynurenic acid, an IDO metabolite, controls TSG-6-mediated immunosuppression of human mesenchymal stem cells. Cell Death Differ. 25, 1209-1223 (2018)

25. Ahasan, M. M. et al. Inflammatory regulation of glucocorticoid metabolism in mesenchymal stromal cells. Arthritis Rheum. 64, 2404-2413 (2012).

26. Tuckermann, J. P., Kleiman, A., McPherson, K. G. \& Reichardt, H. M. Molecular mechanisms of glucocorticoids in the control of inflammation and lymphocyte apoptosis. Crit. Rev. Clin. Lab. Sci. 42, 71-104 (2005).

27. $\mathrm{Wu}, \mathrm{H}$. J. et al. Mesenchymal stem cells modulate albumin-induced renal tubular inflammation and fibrosis. PLOS ONE 9, e90883 (2014).

28. Zhang, L. et al. 11 beta-Hydroxysteroid dehydrogenase 1 inhibition attenuates collagen-induced arthritis. Int. Immunopharmacol. 17, 489-494 (2013).

29. Tomlinson, J. W. et al. The role of 11 beta-hydroxysteroid dehydrogenase 1 in adipogenesis in thyroid-associated ophthalmopathy. J. Clin. Endocrinol. Metab. 95, 398-406 (2010)

30. Thieringer, R. et al. 11 Beta-hydroxysteroid dehydrogenase type 1 is induced in human monocytes upon differentiation to macrophages. J. Immunol. 167 30-35 (2001).

31. Zieg, G., Lack, G., Harbeck, R. J., Gelfand, E. W. \& Leung, D. Y. In vivo effects of glucocorticoids on IgE production. J. Allergy Clin. Immunol. 94, 222-230 (1994).

32. Lee, J. H., Gao, Z. \& Ye, J. Regulation of 11 beta-HSD1 expression during adipose tissue expansion by hypoxia through different activities of NF-kappaB and HIF1alpha. Am. J. Physiol. Endocrinol. Metab. 304, E1035-E1041 (2013).

33. Doig, C. L. et al. TNFalpha-mediated Hsd11b1 binding of NF-kappaB p65 is associated with suppression of 11 beta-HSD1 in muscle. J. Endocrinol. 220 389-396 (2014).

34. Jabara, H. H., Ahern, D. J., Vercelli, D. \& Geha, R. S. Hydrocortisone and IL-4 induce lgE isotype switching in human B cells. J. Immunol. 147, 1557-1560 (1991).

35. Ishii-Yonemoto, T. et al. Glucocorticoid reamplification within cells intensifies NF-kappaB and MAPK signaling and reinforces inflammation in activated preadipocytes. Am. J. Physiol. Endocrinol. Metab. 298, E930-E940 (2010). 